\title{
Inversão 1D de dados Sintéticos do Método Magnetotelúrico
}

\author{
Fernandes,S.C,UFOPA; Piedade,A.A.,UFOPA
}

\section{Copyright 2018, SBGf - Sociedade Brasileira de Geofísica}

Este texto foi preparado para apresentação no VIII Simpósio Brasileiro de Geofísica, em Salinópolis, Pará, Brazil, de 18 a 20 de setembro de 2018. Seu conteúdo foi Revisado Pelo comitê técnico do VIII Simpósio Brasileiro de Geofísica, mas não representa a opinião da SBGf, seus sócios ou membros. A reprodução total ou parcial do conteúdo desse testo para fins comerciais sem o consentimento, por escrito, da Sociedade Brasileira de Geofísica é priobída.

\section{Resumo}

Neste tabalho iremos realizar a modelagem e a inversão de dados sintéticos do método Magnetotelúrico (MT) em um meio de camadas plano horizontais (meio 1D). 0 algorítimo de inversão, não linear, escolhido será o do método de Gauss-Newton com a estratégia de Marquardt. Afim de tornar o problema inverso bem posto, eleminando a ambiguide das soluçãoes, utilizaremos os regularizadores de Suavidade Global (SG) e de Variação Total (VT). Será feito um estudo para identificar camadas alvos mais resistivas e mais condutívas que o seu meio encaixante, utilizando os dois regularizadores anteriormente mencionados.

\section{Introdução}

Os métodos eletromagneticos, são utilizados pelos geofísicos, na investigação das propriedades geoelétricas dos materias que encontram-se abaixo da superfície terrestre, dentre estas propriedades de interesse podemos destacar a resistividade elétrica, a permissividade életrica e a permeabilidade magnética. Na pratica esses métodos se utilizam de correntes que apresentam amplitudes que variam com o tempo, de fontes naurais ou artificiais, de modo a estudar os efeitos indutívos causados em subsuperfície e assim fazer a investigação das propriedades geoelétricas do meio. Dentre os métodos eletromagnéticos, de fontes naturais, está presente o método MagnetoTelúrico (MT), que utiliza de campos eletromagnéticos oriundas sobretudo da perturbação do campo magnético da terra por tempestades solares. Neste trabalho será apresentada a modelagem e a inversão de dados sintéticos do método MT para um meio composto de camadas plano paralelas, nas quais há variação da propriedade física apenas em profundidade (meio 1D). Para a modelagem dos dados seguiremos a metodologia de ondas planas apresentadas em (Rijo, 2004b) já para a inversão utilizaremos o método de Newton para inversão não linear, com a utilização da técnica de Marquardt (Marquardt (1963)) .Pelo fato da inversão de dados geofísicos levar a ambiguidades e instabilidades nos modelos interpretativos estimados, utilizaremos na inversão dois reguralizadores que são o de Suavidade Global e o de Variação Total, de modo a tornar o nosso problema bem posto. A apresentação deste trabalho tem como o objetivo um estudo inicial do método MT, bem como mostrar que essa metodologia pode ser aplicada no estudo de bacias sedimentares, que são os meios geológicos mais próximos do meio hipotético abordado neste trabalho.

\section{Metodologia \\ Dados do métido Magneto Telúrico}

O método magnetotelúrico nos permite estimar a resistividade elétrica em subsuperfície através de medidas em superfície de variações dos campos elétrico e magnético provenientes principalmente da ionosfera terrestre, causados em grande parte por tempestades solares. Estes campos induzem fluxos de correntes elé tricas no interior da Terra, correntes essas chamadas de correntes telúricas. A aquisição deste método é bem prática de de baixo custo, oferecendo informações estruturais e estratigráficas para exploração de áreas extensas. A faixa de frequência dos dados coletados no método varia de $10^{-4} \mathrm{~Hz}$ até aproximadamente $1 \mathrm{kHz}$, sendo as maiores frequências para estudos de objetos mais rasos e as frequências baixas para objetos mais profundos. No método MT a estimativa de resistividade aparente do meio é dado pela seguinte equação

$$
\rho_{a}=\frac{1}{\omega \mu_{o}}\left|\widehat{Z}_{x y}\right|^{2},
$$

que é dada em função da frequência do sinal $\omega$, da permeabilidade magnética do vácuo $\mu_{o}$ e da impedância $\widehat{Z}_{x y}$ do meio em questão, que para o modo de propagação Transversal Magnético (TM) (Rijo, 2004a), é definidada da seguinte maneira

$$
\widehat{Z}_{x y}=\frac{E_{x}}{H_{y}},
$$

sendo $E_{x}$ e $H_{y}$ as componentes dos campos elétrico e magnético, medidos na superfćie terrestre, provenientes da ionosfera e que chegam a superfície da Terra como ondas planas. Os dados que serão utilizados na inversão serão compostos dos valores de resistividade aparente, equação 1 , medidos para uma determinada faixa de frequência. A modelagem dos dados sintéticos de resistividade aparente do método MT foi feita segundo a metodologia descrita em Rijo (2004a).

O método de Gauss-Newton, com a Estratégia de Marquardt, para inversão não linear

Dada as observações geofísicas contidas em um vetor $\mathbf{d}^{o}=\left[d_{1}^{o}, d_{2}^{o}, \ldots, d_{M}^{o}\right]$, e ainda uma função vetorial (modelo matemático), que será considerado não linear, $\mathbf{F}(\mathbf{P})=$ $\left[f_{1}(\mathbf{P}), \ldots, f_{N}(\mathbf{P})\right]$, que é função da frequência $\omega$, das posições de medida $(x, y, z)$, e ainda de um conjunto de parâmetros geofísicos $\mathbf{P}$, pode-se estabelecer a igualdade

$$
\mathbf{d}^{o}=\mathbf{F}(\mathbf{P}) \text {. }
$$

Com o objetivo de encontrar $\mathbf{P}$ que satisfaça 3 , define-se o funcional de ajuste entre os dados observados $\mathbf{d}^{o}$ e os 
estimados por $\mathbf{F}(\mathbf{P})$, utilizando a equação

$$
\phi_{d}(\mathbf{P})=\sum_{i=1}^{M}\left(d_{i}^{o}-f_{i}(\mathbf{P})\right)^{2},
$$

em que $M$ representa o número de observações, $d_{i}^{o}$ a i-ésima componente do vetor de observações e $f_{i}(\mathbf{P})$ a i-ésima componente da função vetorial que reproduz os dados. Na forma matricial pode-se representar este funcional como a norma Euclidiana ,ao quadrado, do vetor de diferênças entre as observações e a função vetorial, ou seja,

$$
\phi_{d}(\mathbf{P})=\left\|\mathbf{d}^{o}-\mathbf{F}(\mathbf{P})\right\|^{2} .
$$

O problema de encontrar $\mathbf{P}$ que minimize 5 é mal-posto, de modo que para torná-lo bem-posto, será utilizado um funcional regularizador, definido aqui genericamente por $\phi_{r}(\mathbf{P})$, que insere informações em relação aos parâmetros $\mathbf{P}$ que se quer estimar. Com isso, o problema a ser resolvido será construído da seguinte forma

$$
\left\{\begin{array}{c}
\text { Minimizar } \phi_{r}(\mathbf{P}), \\
\text { Sujeito a }\|\mathbf{Y}-\mathbf{F}(\mathbf{P})\|^{2}=\delta,
\end{array}\right.
$$

em que $\delta$ representa um erro associado ao modelo matemático não ajustar perfeitamente os dados, devido aos dados conterem ruídos instrumentais ou de ambiente. Resolver este problema significa minimizar o funcional, denominado função objetivo, descrito pela seguinte expressão

$$
\phi_{\alpha}(\mathbf{P})=\phi_{d}(\mathbf{P})+\alpha \phi_{r}(\mathbf{P}),
$$

em que $\alpha$ um escalar positivo, chamado de parâmetro de regularização, que controla a importância da informação inserida pelo funcional regularizador ao processo de inversão. Fazendo uma aproximação de 7 por uma série de Taylor até segunda ordem é possível encontrar

$\phi_{\alpha}(\mathbf{P}) \simeq \phi_{\alpha}\left(\mathbf{P}^{o}\right)+\Delta \mathbf{P}^{T}\left(\mathbf{g}_{d}+\alpha \mathbf{g}_{r}\right)+\frac{1}{2} \Delta \mathbf{P}^{T}\left(\mathbf{H}_{d}+\alpha \mathbf{H}_{r}\right) \Delta \mathbf{P}$.

em que considerou-se

$$
\begin{aligned}
\mathbf{H}_{d} & =\left.\left(\nabla_{\mathbf{P}} \nabla_{\mathbf{P}}^{T} \phi_{d}(\mathbf{P})\right)\right|_{\mathbf{P}=\mathbf{P}^{o}}, \\
\mathbf{H}_{r} & =\left.\left(\nabla_{\mathbf{P}} \nabla_{\mathbf{P}}^{T} \phi_{r}(\mathbf{P})\right)\right|_{\mathbf{P}=\mathbf{P}^{o}}, \\
\mathbf{g}_{d} & =\left.\left(\nabla_{\mathbf{P}} \phi_{d}(\mathbf{P})\right)\right|_{\mathbf{P}=\mathbf{P}^{o},} \\
\mathbf{g}_{r} & =\left.\left(\nabla_{\mathbf{P}} \phi_{r}(\mathbf{P})\right)\right|_{\mathbf{P}=\mathbf{P}^{o}}
\end{aligned}
$$

Logo, para se ter uma estimativa de $\mathbf{P}$ que minimize 8 , se pode aplicar o operador gradiente $\nabla_{\mathbf{P}}$ na mesma e igualar o resultado desta operação ao vetor nulo (Régis, 2000), de modo a obter a seguinte equação normal

$$
\left[2 \mathbf{A}^{T} \mathbf{A}+\alpha \mathbf{H}_{r}\right] \Delta \mathbf{P}=2 \mathbf{A}^{T}\left[\mathbf{d}^{o}-\mathbf{f}\left(\mathbf{P}^{o}\right)\right]-\alpha \mathbf{g}_{r},
$$

em que $\mathbf{H}_{r}$ e $\mathbf{g}_{r}$ serão determinados de acordo com a definição do funcional regularizador. A matriz $\mathbf{A}$, denominada matriz de sensibilidade dos dados em relação aos parâmetros, possui seus componentes definidos pela igualdade

$$
A_{i j}=\frac{\partial f_{i}(\mathbf{P})}{\partial P_{j}} \mid \mathbf{P}^{o}
$$

sendo que neste trabalho a aproximaremos da seguinte maneira

$$
A_{i j}=\left.\frac{\partial f_{i}(\mathbf{P})}{\partial P_{j}}\right|_{\mathbf{P}^{o}} \simeq \frac{f_{i}\left(\mathbf{P} ; P_{j}^{o}+\delta P_{j}^{o}\right)-f_{i}\left(\mathbf{P} ; P_{j}^{o}-\delta P_{j}^{o}\right)}{2 \delta P_{j}^{o}},
$$

sendo $\delta P_{j}^{o}$ uma pequena pertubação do valor do parâmetro $P_{j}^{o}$, que costuma ser um percentual pequeno do valor do mesmo. Neste trabalho será utilizado o valor de $5 \%$. A técnica de Marquardt (Marquardt, 1963), busca garantir a estimativa de um passo sempre na direção descendente do gradiente de $\phi_{\alpha}(\mathbf{P})$. Isto é feito alternando entre a estimativa com o m'etodo de Gauss-Newton, equação 13, e com um pequeno passo na direção descendente do gradiente de $\phi_{\alpha}(\mathbf{P})$, semelhante ao método de gradiente descendente, ou Method of Steepest Descent (Arfken, 1985).

Englobando estas duas formas de buscar um mínimo para a função objetivo, em uma única equação, acrescentase um escalar positivo $\lambda$, denominado parâmetro de Marquardt, em 13, somando-o a diagonal da matriz hessiana dos funcionais, ou seja,

$$
\left[\mathbf{A}_{k}^{T} \mathbf{A}_{k}+\alpha \mathbf{H}_{r k}+\lambda \mathbf{I}\right] \Delta \mathbf{P}_{k}=\mathbf{A}_{k}^{T}\left[\mathbf{d}^{o}-\mathbf{f}\left(\mathbf{P}_{k}\right)\right]-\alpha \mathbf{g}_{r k},
$$

em que $o$ valor de $\lambda$ aumenta ou diminui durante as iterações de modo a deixar a matriz hessiana sempre diagonal dominante.

\section{Regularizador de Suavidade Global}

O vínculo de suavidade global (SG), sujeito ao ajuste dos dados, faz com que o processo convirja para uma solução em que as diferenças entre os valores dos parâmetros sejam mínimas, ou melhor, uma solução em que não há variações abruptas entre os valores dos parâmetros. A representação matemática do funcional SG pode ser escrita como

$$
\phi_{S G}(\mathbf{P})=\sum_{k=1}^{N d}\left|\left(P_{i}-P_{j}\right)_{k}\right|^{2}, \quad i \neq j, \text { i e } j=1,2, \ldots, N p,
$$

ou mesmo na forma matricial

$$
\phi_{S G}(\mathbf{P})=\|\mathbf{S P}\|^{2},
$$

em que $N d$ é o número de diferênças entre os parâmetros, $N p$ o número de parâmetros e $\|\cdot\|^{2}$ a norma dois, ou euclidiana ${ }^{1}$, ao quadrado, do vetor de diferenças SP, onde a matriz $\mathbf{S}$ guarda as informações de como os parâmetros se relacionam. Sua estrutura é de tal forma que, quando multiplicada ao vetor de parâmetros, gere um vetor de diferenças entre parâmetros que se quer relacionar. Para ilustrar a construção de $\mathbf{S}$, tal qual como foi montada para este trabalho, considera-se dois exemplos com seis parâmetros. No primeiro se quer um vetor que guarde a diferença entre os parâmetros adjacentes na horizontal

$$
(\mathbf{S P})^{T}=\left[P_{1}-P_{2}, P_{2}-P_{3}, P_{3}-P_{4}, P_{4}-P_{5}, P_{5}-P_{6}\right] .
$$

no qual o arranjo é mostrado na figura 1.

\footnotetext{
${ }^{1} \mathrm{~A}$ norma $n$ de um vetor pode ser determinada por $\|\mathbf{u}\|_{n}=$ $\left[\sum_{i=1}^{N}\left|u_{i}\right|^{n}\right]^{\frac{1}{n}}$.
} 


\section{$\mathrm{P}_{1} \leftrightarrow \mathrm{P}_{2} \leftrightarrow \mathrm{P}_{3} \leftrightarrow \mathrm{P}_{4} \rightarrow \mathrm{P}_{5} \rightarrow \mathrm{P}_{6}$}

Figure 1: Arranjo dos parâmetros em uma estrutura 1D, que pode ser tanto na vertical como na horizontal.

Para o cado da figura 1, a matriz $\mathbf{S}$ é dada por

$$
\mathbf{S}=\left[\begin{array}{cccccc}
1 & -1 & 0 & 0 & 0 & 0 \\
0 & 1 & -1 & 0 & 0 & 0 \\
0 & 0 & 1 & -1 & 0 & 0 \\
0 & 0 & 0 & 1 & -1 & 0 \\
0 & 0 & 0 & 0 & 1 & -1
\end{array}\right]
$$

A matriz hessiana e o vetor gradiente do funcional de suavidade global, após algumas manipulações matemáticas, serão dados pelas seguintes equações

$$
\begin{aligned}
\mathbf{g}_{S G} & =\nabla_{\mathbf{P}} \phi_{S}(\mathbf{P})=2 \mathbf{S}^{T} \mathbf{S P} \\
\mathbf{H}_{S G} & =\nabla_{\mathbf{P}} \nabla_{\mathbf{P}}^{T} \phi_{S}(\mathbf{P})=2 \mathbf{S}^{T} \mathbf{S} .
\end{aligned}
$$

\section{Regularizador de Variação Total}

O funcional de variação total (VT) é o mais indicado quando se quer estimar soluções que hajam descontinuidades nos valores de alguns parâmetros, enquanto que para outros se tenham apenas variações suaves entre seus valores. Pode-se definir o funcional do vínculo VT (Lima et al., 2011) como a norma um do vetor de diferenças entre os parâmetros, ou mesmo,

$\phi_{V T}(\mathbf{P})=\sum_{k=1}^{N d}\left|\left(P_{i}-P_{j}\right)_{k}\right|=\|\mathbf{S P}\|, \quad i \neq j$, i e $j=1,2, \ldots, N p$,

em que $\mathbf{S}$, em que $\mathbf{S}$ tem a forma de 20. Definido o funcional, deve-se obter seu vetor gradiente bem como sua matriz hessiana, no entanto o funcional 23 não possui derivada quando $P_{i}=P_{j}$. Este problema é contornado quando se faz a aproximação

$$
\left|\left(P_{i}-P_{j}\right)_{k}\right| \simeq\left[\left(P_{i}-P_{j}\right)_{k}^{2}+\beta\right]^{\frac{1}{2}}
$$

sendo $\beta$ um escalar real de valores pequenos, porém maiores que zero. O critério para a escolha do valor dessa constante é testar o menor valor que combinado com o valor ótimo do parâmetro de regularização, possibilite uma soluç estável para o problema. O valor de $\beta$ bem como o valor do parâmetro de regularização $\alpha$ não se modificam durente o processo iterativo, na busca de uma solução. Reescrevendo 23 como

$$
\phi_{V T}(\mathbf{P})=\sum_{k=1}^{N d}\left[\left(P_{i}-P_{j}\right)_{k}^{2}+\beta\right]^{\frac{1}{2}},
$$

pode-se determinar a matriz hessiana e o vetor gradiente deste (Lima et al., 2011), de modo a encontrar

$$
\mathbf{G}_{V T}=\mathbf{S}^{T} \mathbf{q}, \quad q_{k}=\frac{\left(P_{i}-P_{j}\right)}{\left[\left(P_{i}-P_{j}\right)_{k}^{2}+\beta\right]^{\frac{1}{2}}}
$$

bem como

$$
\mathbf{H}_{V T}=\mathbf{S}^{T} \mathbf{Q S}
$$

sendo $Q$ uma matriz diagonal, com seus valores não nulos dados por

$$
Q_{k k}=\frac{\beta}{\left[\left(P_{i}-P_{j}\right)_{k}^{2}+\beta\right]^{\frac{3}{2}}}
$$

\section{Resultados}

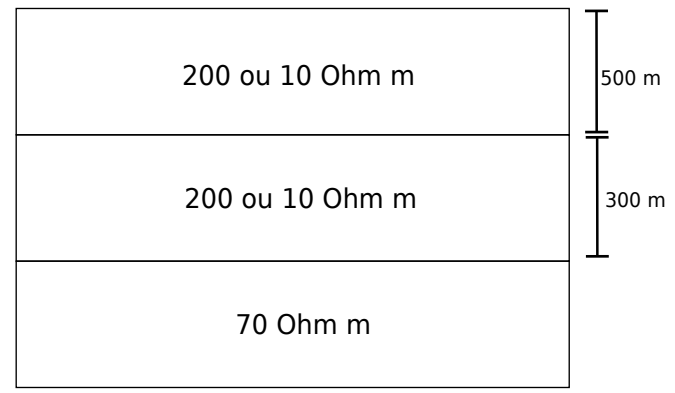

Figure 2: Modelo que gerou os dados dos resultados das figuras 3 e 4 .
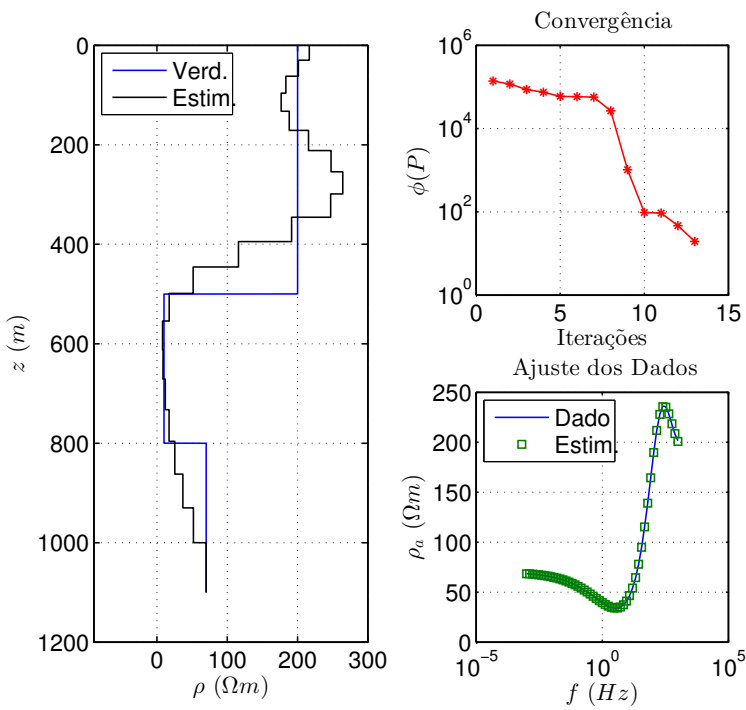

Figure 3: Resultado com o vínculo SG para o modelo da figura 2 com a camada central mais condutiva.

Inicialmente, serão gerados resultados para os dois modelos de camadas mostrados na figuras 2 e 5, usando apenas o regularizador de Suavidade Global. Para os dois modelos, mudaremos as resistividade das duas primeiras camadas, afim de estudar o comportamento das soluções quando se tem alvos mais condutívos ou resistivos. O primeiro modelo consiste de três camadas, sendo a última o embasamento. A primeira camada possui uma espessura de $500 \mathrm{~m}$ e resistividade de 200 $\Omega m$, a segunda $300 \mathrm{~m}$ e resistividade de $10 \Omega m$ e o embasamento $70 \Omega \mathrm{m}$. Em todos os modelos gerou-se dados de resistividade aparente na faixa de frequência de 

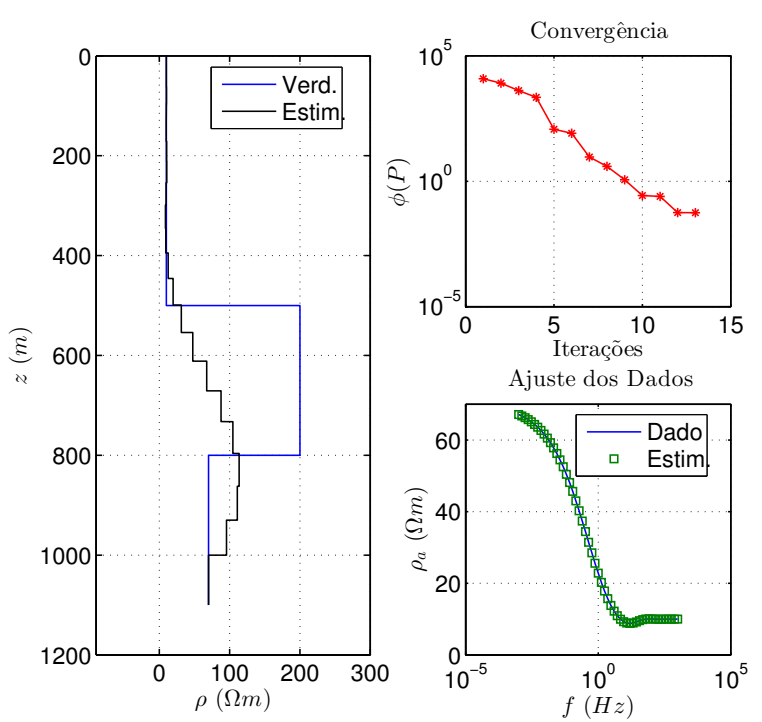

Figure 4: Resultado com o vínculo SG para o modelo da figura 2 com a camada central mais resistiva.

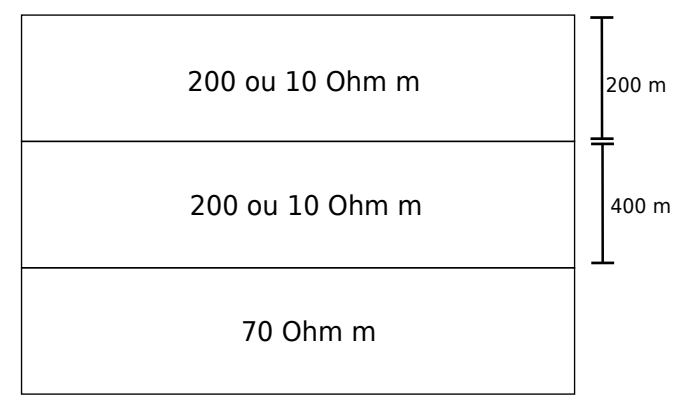

Figure 5: Modelo que gerou os dados dos resultados das figuras 6 e 7 .
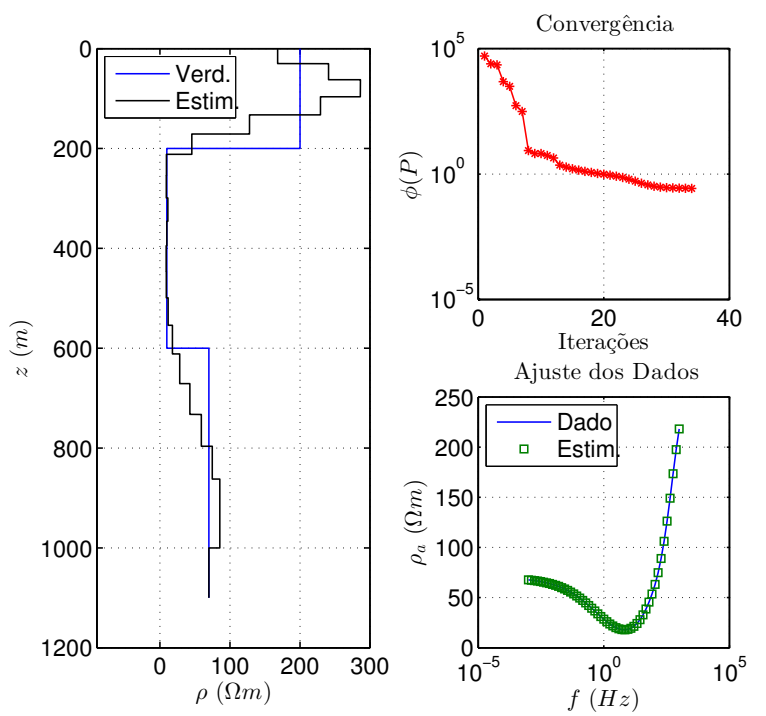

Figure 6: Resultado com o vínculo SG para o modelo da figura $5 \mathrm{com}$ a camada central mais condutiva.
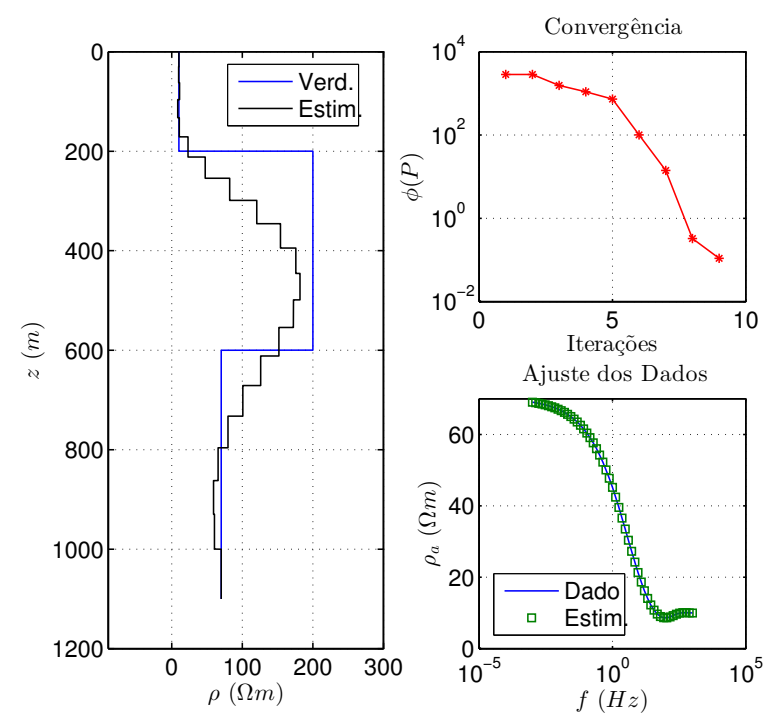

Figure 7: Resultado com o vínculo SG para o modelo da figura 5 com a camada central mais resistiva.

$0.001 \mathrm{~Hz}$ a $1 \mathrm{kHz}$. O modelo interpretativo usado para gerar os resultados de inversão, em todos os modelos, consiste de 20 camadas de espessuras crescentes, sendo a primeira com 30 metros de espessura. Para todos os modelos invertidos considerou-se, como um modelo inicial, a resistividade de todas as camadas do modelo interpretativo iguais a $50 \Omega \mathrm{m}$. Nas figuras 3 e 4 utilizouse o vínculo SG para tentar recuperar o primeiro modelo, obtendo bons resultados. No resultado da figura 3 o parâmetro de regularização usado foi $\alpha=10$ e no da figura 4 utilizou-se $\alpha=10^{-1}$. Seguidamente, gerou-se dados de resistividade aparente para o modelo mostrado na figura 5 , invertendo a resistividade da primeira e da segunda camada com as resistividades mostradas na própria figura. Os resultados de inversão com os vínculo SG podem ser visualizados nas figuras 6 e 7, ambos utilizando o parâmetro de regularização $\alpha=10^{-1}$.

O algorítimo que irá implementar o regularizador de variação total ainda está em fase de construção, daí a não inclusão de resultados com o mesmo.

\section{Conclusões}

Neste trabalho teve-se como objetivo a análise numérica da modelagem e da inversão de dados sintéticos do Método Magnetotelúrico, em meios estratificados 1D. Por meio dos modelos interpretativos resuperados podese observar excelentes resultados com o algorítomo de inversão desenvolvido. Observou-se com os dois modelos que camadas mais resistívas são melhores recuperadas quanto mais rasas e mais espessas forem, enquanto que as camadas mais condutívas em ambos os modelos foram bem identificadas, o que é de se esperar por se tratar o MT de um método indutivo. Por se tratar de uma inversão 1D o custo computacional é bastante baixo o que viabiliza e muito a sua utilizacão com dados reais em modelos mais realísticos. 


\section{References}

Arfken, G., 1985, Mathematical methods for physicists, third ed.: Academic Press.

Lima, W. A., C. M. Martins, V. C. F. Barbosa, and J. B. C. Silva, 2011, Total variation regularization for depth-tobasement estimate: Part 1 - mathematical details and applications: Geophysics, 76, I1-I12.

Marquardt, D. W., 1963, An algorithm for least-squares estimation of nonlinear parameters: SIAM Journal on Applied Mathematics, 11, 431-441.

Régis, C. R. T., 2000, Inversão vinculada de dados de emap e mt-1d anisotrópico: Tese de doutorado, Programa de Pós-Graduação em Geofísica, Universidade Federal do Pará.

Rijo, L., 2004a, Eletrical geophysics 1-d earth modeling. , 2004b, Eletromagnetismo com matemática. 